\title{
Echo-enabled harmonic generation free electron laser
}

\author{
Dao Xiang and Gennady Stupakov \\ SLAC National Accelerator Laboratory, Menlo Park, California 94025, USA
}

(Received 2 December 2008; published 4 March 2009)

\begin{abstract}
In this paper, we systematically study the echo-enabled harmonic generation (EEHG) free electron laser (FEL). The EEHG FEL uses two modulators in combination with two dispersion sections that allow one to generate in the beam a high harmonic density modulation starting with a relatively small initial energy modulation of the beam. After presenting an analytical theory of the phenomenon, we address several practically important issues, such as the effect of incoherent synchrotron radiation in the dispersion sections, and the beam transverse size effect in the modulator. Using a representative realistic set of beam parameters, we show how the EEHG scheme enhances the FEL performance and allows one to generate a fully (both longitudinally and transversely) coherent radiation. As an example, we demonstrate that $5 \mathrm{~nm}$ coherent soft x rays with GW peak power can be generated directly from the $240 \mathrm{~nm}$ seeding laser using the proposed EEHG scheme.
\end{abstract}

DOI: 10.1103/PhysRevSTAB.12.030702

PACS numbers: 41.60.Cr

\section{INTRODUCTION}

There has been continually growing interest in generating coherent and powerful short wavelength radiation using the free electron laser (FEL) scheme, as reflected by the many proposals and funded projects worldwide [1]. In the nanometer and subnanometer wavelengths, the two leading candidates are self-amplified spontaneous emission (SASE) configuration [2,3] and the high gain harmonic generation (HGHG) scheme [4,5]. Since the SASE FEL starts from electron beam shot noise, the output of SASE FEL typically has limited temporal coherence and relatively large shot-to-shot fluctuations in both the power and the spectrum. An alternative to SASE configuration is the HGHG scheme that allows generation of temporally coherent radiation by using up-frequency conversion of a high-power seeding signal.

In the classic HGHG scheme [5], the electron beam is first energy modulated with a seed laser in the undulator (modulator) and then sent through a dispersion region which converts the energy modulation into a density modulation. The density modulated beam is then sent through the second undulator (radiator) tuned at some harmonic of the seed laser. The up-frequency conversion efficiency for this classic HGHG scheme is relatively low: generation of the $n$th harmonic of the seed laser requires the energy modulation amplitude approximately equal to $n$ times the slice energy spread of the beam. Because a considerable increase of the slice energy spread would significantly degrade the lasing process in the radiator, the harmonic numbers $n$ used in the classic HGHG scheme are typically no larger than 6 . In order to generate coherent soft $\mathrm{x}$ rays with a wavelength in the range of a few nanometers using an ultraviolet (UV) wavelength seeding laser with the wavelength $\sim 200 \mathrm{~nm}$, multiple stages of the classic HGHG FEL are to be used [6].
In order to get higher harmonics while keeping the energy spread growth within an acceptable level, a double-undulator HGHG scheme was recently suggested [7] where the modulator is subdivided into two pieces with a $\pi$ phase shifter between them. This allows for generation of substantial bunching at higher harmonics while simultaneously limiting the growth of the energy spread. However, the double-undulator HGHG scheme still requires a high laser power and small beam slice energy spread, which may limit its practical applications.

Recently one of the authors (G.S.) proposed a new method for generation of high harmonics using the beam echo effect [8]. The echo scheme has a remarkable upfrequency conversion efficiency and allows for generation of high harmonics with a relatively small energy modulation. The echo scheme uses two modulators and two dispersion sections. In general, the frequencies of the first, $\omega_{1}$, and the second, $\omega_{2}$, modulators can be different. The beam modulation is observed at the wavelength $2 \pi / k_{E}$, where $c k_{E}=n \omega_{1}+m \omega_{2}$, with $n$ and $m$ integer numbers. The first dispersion section is chosen to be strong enough, so that the energy and the density modulations induced in the first modulator are macroscopically smeared due to the slippage effect. At the same time, this smearing introduces a complicated fine structure into the phase space of the beam. The echo then occurs as a recoherence effect caused by the mixing of the correlations between the modulation in the second modulator and the structures imprinted onto the phase space by the combined effect of the first modulator and the first dispersion section. The key advantage of the echo scheme is that the amplitude of high harmonics of the echo is a slow decaying function of the integer numbers $n$ and $m$.

In this paper we systematically study the echo-enabled harmonic generation (EEHG) FEL. We first introduce the principles of the EEHG FEL in Sec. II. We then proceed to 
present the physical mechanism of the echo effect in Sec. III. The issues that may affect the performance of EEHG FEL are studied in Sec. IV. With realistic beam parameters, we show in Sec. V that $5 \mathrm{~nm}$ coherent soft $\mathrm{x}$ ray with GW peak power can be generated directly from the $240 \mathrm{~nm}$ seeding laser using the EEHG scheme. The conclusions are summarized in Sec. VI.

\section{PRINCIPLES OF EEHG FEL}

The schematic of the EEHG FEL is shown in Fig. 1. The EEHG FEL consists of two modulators, two dispersion sections, and one radiator. Similar to the classic HGHG scheme, a laser pulse is used to modulate the beam energy in the first undulator (modulator 1). If the bunch length is much larger than the wavelength of the modulation, we can neglect the variation of the beam current within the bunch and assume a longitudinally uniform beam.

Following the notation of Ref. [8], we assume an initial Gaussian beam energy distribution with an average energy $E_{0}$ and the rms energy spread $\sigma_{E}$, and use the variable $p=$ $\left(E-E_{0}\right) / \sigma_{E}$ for the dimensionless energy deviation of a particle. The initial longitudinal phase space distribution can then be written as $f_{0}(p)=N_{0}(2 \pi)^{-1 / 2} e^{-p^{2} / 2}$, where $N_{0}$ is the number of electrons per unit length of the beam.

After passage through the first undulator, the beam energy is modulated with the amplitude $\Delta E_{1}$, so that the final dimensionless energy deviation $p^{\prime}$ is related to the initial one $p$ by the equation $p^{\prime}=p+A_{1} \sin \left(k_{1} z\right)$, where $A_{1}=\Delta E_{1} / \sigma_{E}, k_{1}=\omega_{1} / c$, and $z$ is the longitudinal coordinate in the beam. The distribution function after the interaction with the laser becomes $f_{1}(\zeta, p)=$ $N_{0}(2 \pi)^{-1 / 2} \exp \left[-\left(p-A_{1} \sin \zeta\right)^{2} / 2\right]$, where we now use the dimensionless variable $\zeta=k_{1} z$. Sending then the beam through the first dispersion section with the dispersive strength $R_{56}^{(1)}$ converts the longitudinal coordinate $z$ into $z^{\prime}, z^{\prime}=z+R_{56}^{(1)} p \sigma_{E} / E_{0}$ (where $p$ now refers to the value at the entrance to the first dispersion section), and

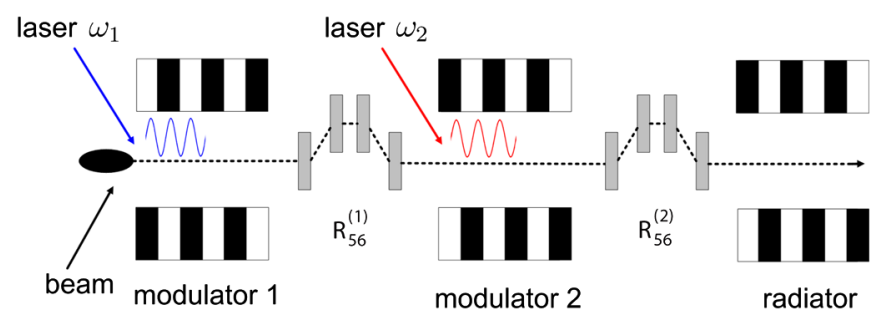

FIG. 1. (Color) Schematic of the EEHG FEL. The beam energy is modulated in the first undulator (modulator 1) tuned at frequency $\omega_{1}$ due to the interaction with the first laser beam. After passing through the first dispersion section with $R_{56}^{(1)}$, the beam energy is then modulated in the second undulator (modulator 2) tuned at frequency $\omega_{2}$ due to the interaction with the second laser beam. The beam passes through the second dispersion section $R_{56}^{(2)}$ and emits radiation in the last undulator (radiator). makes the distribution function

$$
f_{2}(\zeta, p)=\frac{N_{0}}{\sqrt{2 \pi}} \exp \left[-\frac{1}{2}\left[p-A_{1} \sin \left(\zeta-B_{1} p\right)\right]^{2}\right],
$$

where $B_{1}=R_{56}^{(1)} k_{1} \sigma_{E} / E_{0}$.

The final distribution function at the exit from the second dispersion section can be easily found by applying consecutively two more transformations to (1), similar to the derivation outlined above. The first of these two transformations corresponding to the modulation of the beam energy with dimensionless amplitude $A_{2}$ is $p^{\prime}=$ $p+A_{2} \sin \left(k_{2} z+\phi\right)$, where $\phi$ is a phase of the second laser beam; and the second one corresponding to the passage through the second dispersive element is $z^{\prime}=z+$ $p R_{56}^{(2)} \sigma_{E} / E_{0}$ (where $p$ now refers to the value at the entrance to the second dispersion section). The resulting final distribution function $f_{f}$ is

$$
\begin{aligned}
f_{f}(\zeta, p)= & \frac{N_{0}}{\sqrt{2 \pi}} \exp \left[-\frac{1}{2}\left\{p-A_{2} \sin \left(K \zeta-K B_{2} p+\phi\right)\right.\right. \\
& -A_{1} \sin \left[\zeta-\left(B_{1}+B_{2}\right) p\right. \\
& \left.\left.\left.+A_{2} B_{1} \sin \left(K \zeta-K B_{2} p+\phi\right)\right]\right\}^{2}\right]
\end{aligned}
$$

where $B_{2}=R_{56}^{(2)} k_{1} \sigma_{E} / E_{0}$, and $K=k_{2} / k_{1}$.

Integration of this formula over $p$ gives the beam density $N$ as a function of $\zeta, N(\zeta)=\int_{-\infty}^{\infty} d p f_{f}(\zeta, p)$. We define the bunching factor $b$ as

$$
b=\frac{1}{N_{0}}\left|\left\langle e^{-i a \zeta} N(\zeta)\right\rangle\right|,
$$

where $a$ is a number, and the brackets denote averaging over the coordinate $\zeta$. As we show in the Appendix, the bunching factor is not zero only if

$$
a=n+K m,
$$

which means presence of a modulation with the wave number $k_{E} \equiv a k_{1}=n k_{1}+m k_{2}$, where $n$ and $m$ are integer numbers. Note that $n$ and $m$ can be either positive or negative, with a negative $k_{E}$ meaning a modulation with a wavelength $2 \pi /\left|k_{E}\right|$. Using the notation $b_{n, m}$ for the bunching factor (3) with $a$ defined by Eq. (4), we find in the Appendix

$$
\begin{aligned}
b_{n, m}= & \mid e^{-(1 / 2)\left[n B_{1}+(K m+n) B_{2}\right]^{2}} J_{m}\left[-(K m+n) A_{2} B_{2}\right] \\
& \times J_{n}\left\{-A_{1}\left[n B_{1}+(K m+n) B_{2}\right]\right\} \mid .
\end{aligned}
$$

We will now show how to choose the dimensionless parameters $A_{1}, A_{2}, B_{1}$, and $B_{2}$ to maximize the absolute value of the bunching factor $b_{n, m}$ for given $n, m$, and the ratio of the frequencies $K$. Analysis shows that the bunching factor attains its maximum when $n= \pm 1$ and decreases as the absolute value of $n$ increases. As we will see below, in order for $B_{1}$ and $B_{2}$ to have the same sign 


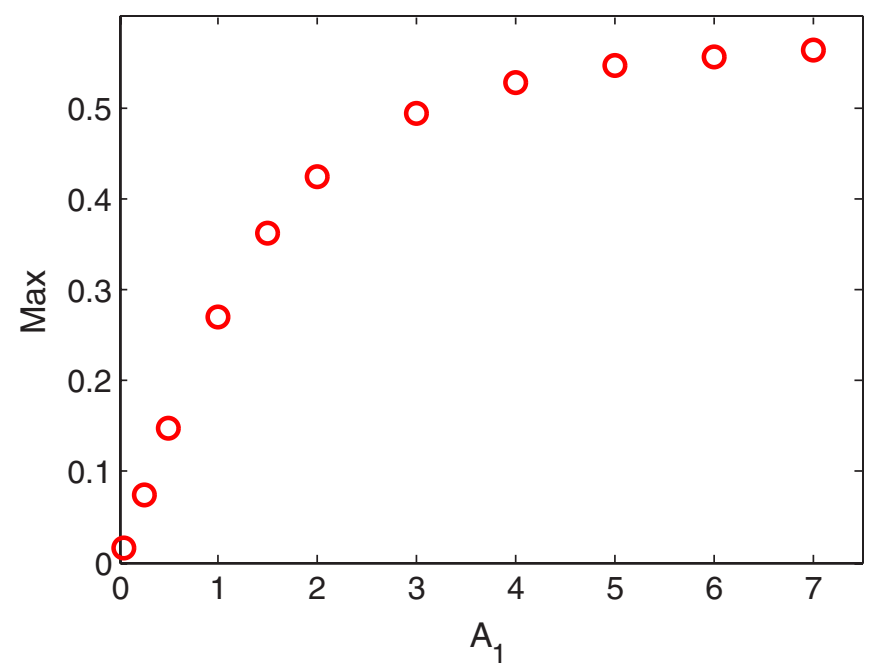

FIG. 2. (Color) Maximal value of $J_{1}\left(A_{1} \xi\right) e^{-\xi^{2} / 2}$ as a function of parameter $A_{1}$.

(which means that one can use either two chicanes or two doglegs as dispersion elements), $n$ and $m$ need to have opposite signs. So we will limit our consideration by the case $n=-1$ and $m>0$ only. Other cases can be studies in a similar way. We start from

$$
\begin{aligned}
b_{-1, m}= & \mid J_{m}\left[(K m-1) A_{2} B_{2}\right] J_{1}\left\{A_{1}\left[B_{1}-(K m-1) B_{2}\right]\right\} \\
& \times e^{-(1 / 2)\left[B_{1}-(K m-1) B_{2}\right]^{2}} \mid .
\end{aligned}
$$

Note that for $m>4$, the maximal value of the Bessel function $J_{m}$ is about $0.67 / \mathrm{m}^{1 / 3}$ and is achieved when its argument is equal to $m+0.81 m^{1 / 3}$ [9]. So in order to maximize the $J_{m}$ factor in Eq. (6), we choose

$$
(K m-1) A_{2} B_{2}=m+0.81 m^{1 / 3} .
$$

To find the parameters that maximize the product $J_{1}\left\{A_{1}\left[B_{1}-(K m-1) B_{2}\right]\right\} e^{-(1 / 2)\left[B_{1}-(K m-1) B_{2}\right]^{2}}$, we introduce the variable $\xi=B_{1}-(K m-1) B_{2}$, differentiate with respect to $\xi$, and set the derivative equal to zero

$$
A_{1}\left[J_{0}\left(A_{1} \xi\right)-J_{2}\left(A_{1} \xi\right)\right]=2 \xi J_{1}\left(A_{1} \xi\right) .
$$

It is easy to find that among the infinite number of roots of Eq. (8), only two roots that have minimal absolute value maximize the expression. The bunching factor is maximized when Eqs. (7) and (8) are both satisfied. The maximal value of $J_{1}\left(A_{1} \xi\right) e^{-\xi^{2} / 2}$ is plotted in Fig. 2 as a function of parameter $A_{1}$. From Fig. 2 we see that the maximal value of this function increases linearly with $A_{1}$ when $A_{1}$ is smaller than 2 . When $A_{1}$ becomes larger than 3 , the growth of the maximal values slows down, and when $A_{1}$ tends to infinity, the maximal value approaches 0.58 . In this limit, as it follows from Eq. (6), the maximal bunching factor becomes (assuming $m>4$ )

$$
\left|b_{-1, m}\right| \approx \frac{0.39}{m^{1 / 3}} .
$$

It should be pointed out that the bunching factor will decrease if the dispersion strengths are not at the optimized values [8]. In practical design of an EEHG FEL, efforts should be made to make the energy modulation amplitudes and the dispersion strengths close to the optimized values.

\section{PHYSICAL MECHANISM OF THE ECHO EFFECT}

To illustrate the physical mechanism behind the echo effect, we first recall the mechanism of the classic HGHG scheme. For simplicity, we consider a longitudinally uniform beam, assume a one-dimensional phase space $p-z$, and neglect the transverse emittance and the finite beam size effects. The initial longitudinal phase space of the beam is shown in Fig. 3(a). After the first modulator, the longitudinal phase space evolves to Fig. 3(b) (we assumed here the relative amplitude of the energy modulation $A=$ $3)$. In the classic HGHG scheme, the dispersion is chosen to approximately satisfy $R_{56} \Delta E / E \approx \lambda / 4$ (assuming $\Delta E \gg \sigma_{E}$ ), where $\lambda$ is the laser wavelength, so that the electrons in the peak and those in the valley move towards the zero crossing by $\lambda / 4$ after the dispersion, see Fig. 3(c). With this optimized dispersion strength, the energy modulation is converted to current modulation and the localized current bumps contain higher harmonic components.
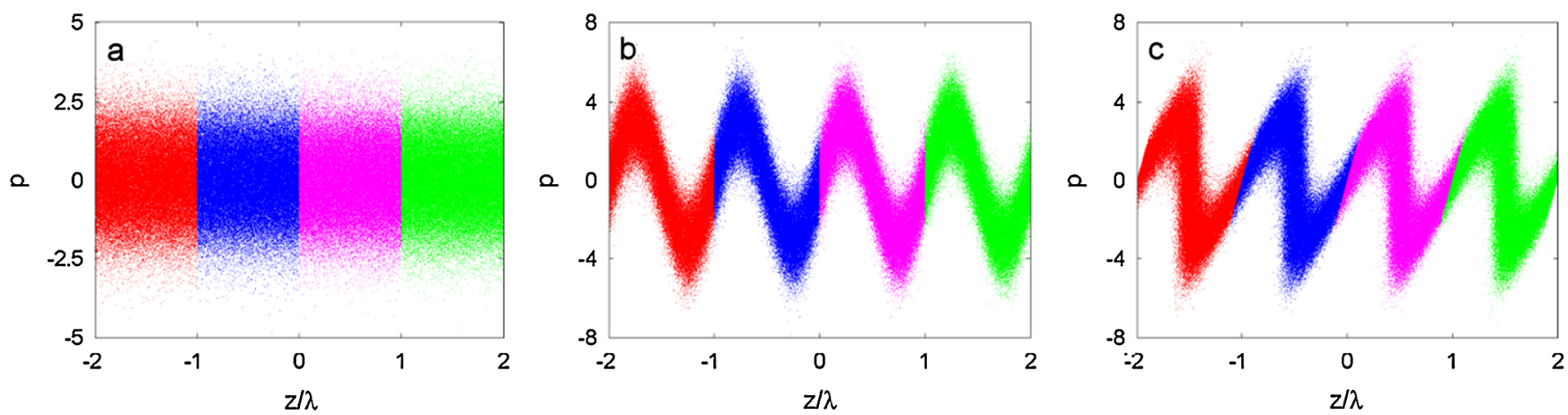

FIG. 3. (Color) The phase space evolution in the classic HGHG scheme. Different colors indicate four regions of unit laser wavelength in the initial phase space of the beam. 

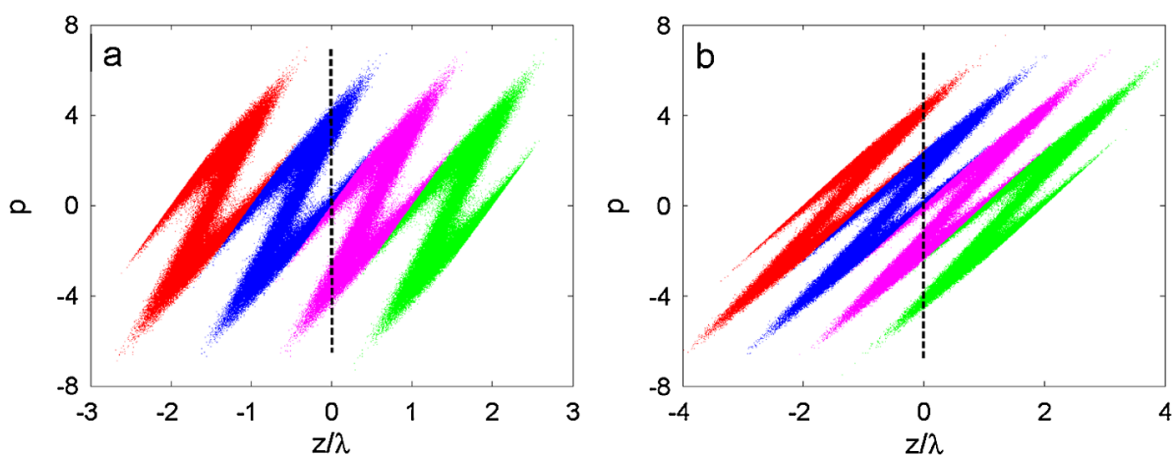

FIG. 4. (Color) Longitudinal phase space after beam passing through a dispersion section with strength (a) $R_{56} \Delta E / E=\lambda$, and (b) $R_{56} \Delta E / E=2 \lambda$.

As we will see below, the echo modulation scheme utilizes much larger dispersion strength in dispersion section 1. When the dispersion strength is increased, at a given longitudinal position $z$, one observes particles that arrive from different wavelength ranges of the initial phase space. This results in a complicated structure in the phase space illustrated by Fig. 4. Looking at the coordinate $z=0$ in Figs. 4(a) and 4(b), we see that the beam at this position is split into three and five beamlets, respectively, separated in energy. From Eq. (1), we find that the energy distribution of the electrons at $z=0$ can be written as

$$
f_{2}(0, p)=\frac{N_{0}}{\sqrt{2 \pi}} \exp \left[-\frac{1}{2}\left[p+A_{1} \sin \left(B_{1} p\right)\right]^{2}\right] .
$$

This energy distribution function (normalized by $N_{0}$ ) for various values of $B_{1}$ is shown in Fig. 5. As follows from this figure, an increase of $R_{56}$ generates more energy bands in the phase space. The presence of the separated energy bands at the same longitudinal position indeed allows for the generation of high harmonics of the density modulation

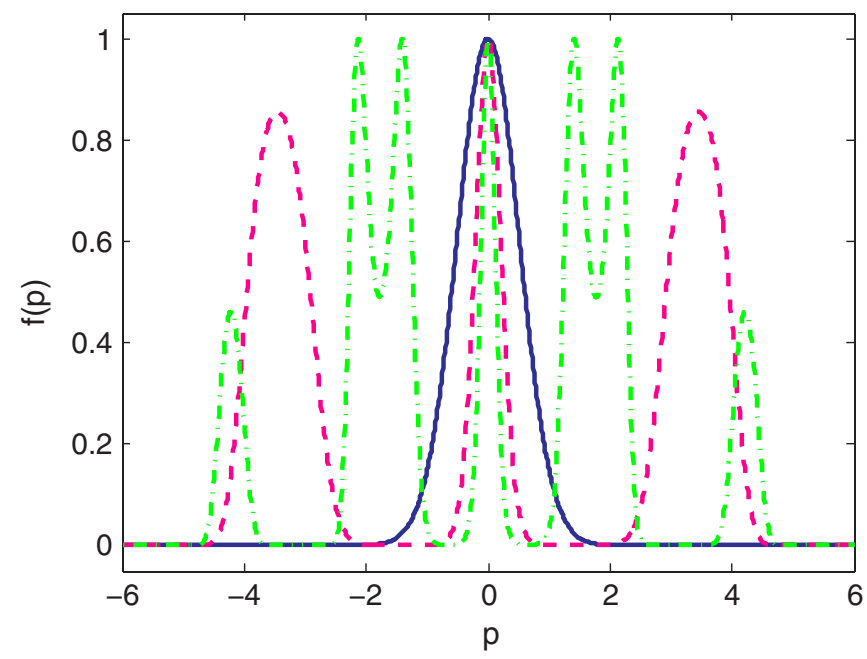

FIG. 5. (Color) Energy distribution at $z=0$ for various values of $R_{56}$. Solid blue curve: $R_{56} \Delta E / E=\lambda / 4$; dashed magenta line: $R_{56} \Delta E / E=\lambda$; and dash-dotted green line: $R_{56} \Delta E / E=2 \lambda$. after these separated energy bands are again modulated in the second modulator and then converted to separated density bands in the second dispersion section.

To illustrate a possible application of EEHG in a system with realistic parameters, we take the nominal parameters of the Fermi@Elettra FEL project [10] and show below how the 24th harmonic of the seed laser can be generated with a relatively small energy modulation using the EEHG scheme. The electron beam energy of the Fermi@Elettra FEL is $1.2 \mathrm{GeV}$ and the slice energy spread is $150 \mathrm{keV}$. We assume the wavelength of the seed laser $240 \mathrm{~nm}$ with the frequency of the first and the second modulators equal, $\omega_{1}=\omega_{2}$. The energy modulation amplitudes in modulator 1 and modulator 2 are chosen to be $A_{1}=3$ and $A_{2}=1$, respectively. Note that the energy modulation amplitude in our example is much smaller than the nominal value of the modulation amplitude in the Fermi@Elettra project. The optimized dispersion strengths obtained from Eqs. (7) and (8) that maximize the bunching factor for the 24th harmonic are found to be $B_{1}=26.83$ and $B_{2}=1.14$, corresponding to $R_{56}^{(1)}=8.20 \mathrm{~mm}$ and $R_{56}^{(2)}=0.35 \mathrm{~mm}$, respectively. The maximal displacement change of the electrons after passing through the first dispersion section is about $A_{1} B_{1} / k \approx 13 \lambda$ that is small compared to the overall bunch length. So the assumption of a longitudinal uniform beam in Sec. II is well justified.

The longitudinal phase space of the beam after passing through the dispersion section 1 is shown in Fig. 6(a) where the presence of separated energy bands is clearly seen. A second laser with the same wavelength is used to modulate the beam energy in modulator 2 . At the exit of modulator 2, the longitudinal phase space is shown in Fig. 6(b). It is worth pointing out that the inherent energy spread of each energy band is much smaller than that of the whole beam. After passing through the dispersion section 2, the longitudinal phase space evolves to that in Fig. 6(c), where we clearly see that the energy modulation for each energy band is converted to a density modulation. When projected onto the $z$ axis, the phase space of Fig. 6(c) clearly shows a current modulation, shown in Fig. 6(d), with approximately 24 spikes in one wavelength region, which indicates 

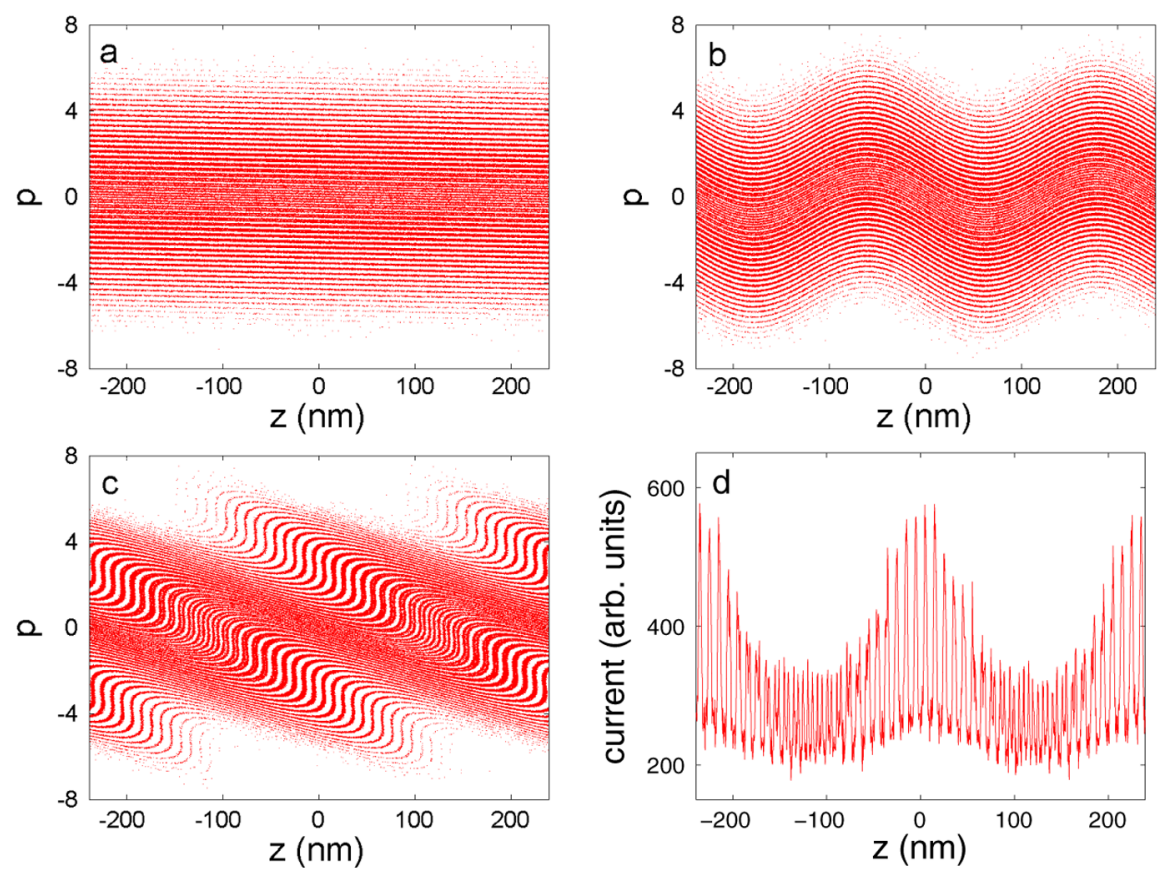

FIG. 6. (Color) Longitudinal phase space evolution in the EEHG scheme. (a) Phase space at the exit of dispersion section 1. (b) Phase space at the exit of modulator 2. (c) Phase space at the exit of dispersion section 2. (d) Current distribution at the exit of dispersion section 2 .

a presence of the 24th harmonic of the seed laser. Fourier transform of the current distribution in Fig. 6(d) gives the bunching factor at various harmonic numbers shown in Fig. 7. For convenience of comparison, the bunching factor for the optimized classic HGHG scheme with the same energy modulation amplitude is also shown in Fig. 7. From Fig. 7 we see that for the classic HGHG scheme the bunching factor exponentially decreases as the harmonic number increases. However, for the echo scheme, we can

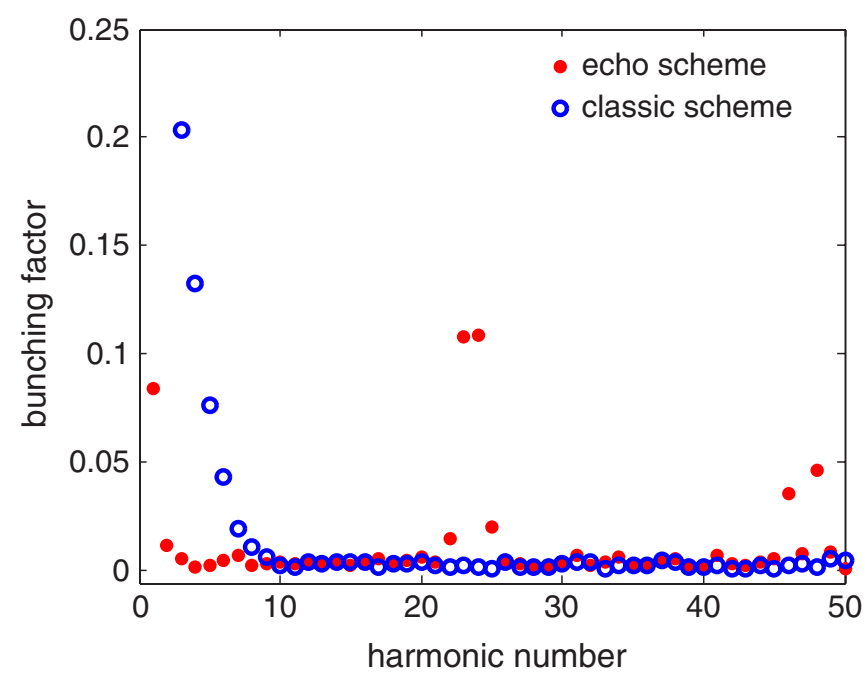

FIG. 7. (Color) Comparison of the bunching factors from the EEHG scheme (red dots) and that from the classic HGHG scheme (blue circles). intentionally maximize the bunching factor for some specific harmonic number while most of the other harmonic components are effectively suppressed. In addition to generating the 24th harmonic, the echo signal also contains noticeable components for the 48th and 72th harmonic.

\section{ISSUES AFFECTING PERFORMANCE OF EEHG FEL}

The unique feature of EEHG FEL is utilization of two laser beams and relatively large dispersion strength for the dispersion section 1 . We will discuss in this section such issues as effect of incoherent and coherent synchrotron radiation (ISR and CSR, respectively), as well as the transverse size of the laser beams, that may affect the performance of the FEL.

For the classic HGHG scheme, CSR and ISR effects in the dispersion section are generally neglected, due to the fact that the $R_{56}$ is relatively small. For the EEHG case, the dispersion strength is approximately 1 or 2 orders of magnitude larger, and these effects may play important roles in generation of the beam modulation. Quantum fluctuations in the process of incoherent synchrotron radiation lead to diffusion in energy. If the rms value of the energy spread caused by this diffusion exceeds the spacing of two adjacent energy bands, it may result in the overlapping of the bands, which will smear the fine structures of the longitudinal phase space and thus degrade the EEHG performances. The $z$-dependent CSR wake introduces an additional energy modulation inside the dispersive section, 
which may result both in emittance growth of the beam and distortion of the echo modulation.

To find the spacing between the adjacent energy bands, we take the derivative of Eq. (10) and equate it to zero. We have

$$
\left[1+A_{1} B_{1} \cos \left(B_{1} p\right)\right]\left[p+A_{1} \sin \left(B_{1} p\right)\right]=0 .
$$

A detailed analysis shows that Eq. (10) attains its minimum when $1+A_{1} B_{1} \cos \left(B_{1} p\right)=0$, and it attains its maximum when $p+A_{1} \sin \left(B_{1} p\right)=0$. These equations can be easily solved numerically. As an example, we consider parameters of a modulator used in the previous section to maximize the 24th harmonic: $A_{1}=3$ and $B_{1}=26.83$. Numerically solving Eq. (11), we find that the spacing varies with $p$ : in the central region of the phase space where $p$ is small, the spacing between adjacent energy bands is about $0.09 \sigma_{E}$, and it increases to about $0.25 \sigma_{E}$ for large $p$. So we can expect that the fine structures in the central part of the phase space where $p$ is small are most vulnerable to the energy diffusion caused by ISR. Assuming $\sigma_{E}=150 \mathrm{keV}$, we find that the smallest spacing between the energy bands is about $13 \mathrm{keV}$.

To compare this spacing with the energy spread introduced by passing a length $L$ in a bend with the bending radius $\rho$, we use the following formula [11]:

$$
\left.\Delta \sigma_{E}^{2}\right|_{\mathrm{ISR}}=\frac{55 e^{2} \hbar c}{48 \sqrt{3}} \frac{L}{\rho^{3}} \gamma^{7},
$$

where $\gamma$ is the relativistic factor. We calculated the ISR induced energy spread for a very compact dispersion section design consisting of a symmetric 4-dipole chicane with a dispersion strength of $8.20 \mathrm{~mm}$. The length of each dipole is $20 \mathrm{~cm}$ and the distances between the first and second dipoles and that between the third and fourth dipoles are both $25 \mathrm{~cm}$. The distance between the second and third dipoles is $10 \mathrm{~cm}$ and the bending angle is about 5.9 degrees. We found that the ISR induced energy spread is about $2.9 \mathrm{keV}$, which is not negligible as compared to the spacing of the separated energy bands. To find out the degradation of the bunching process caused by ISR we carried out simulations with the computer code ELEGANT [12]. The effect of transverse emittance and second order elements of the transport matrix were included in the simulation. When the parameters of the laser and dispersion strength were optimized to maximize the bunching factor for the 24th harmonic, we found out that turning on the ISR tracking option in the code decreased the bunching factor by about $20 \%$ compared to the case when the ISR option was turned off. The simulation results also imply that the emittance and the second order effect has no noticeable effect on the smearing of the longitudinal phase space.

It is worth pointing out that the diffusion caused by ISR strongly depends on the design of the dispersion section. In the example above we used a very compact dispersion section to be able to demonstrate the ISR effect. In practical design, for a given dispersion strength, one can increase the spacing between the dipoles and thus decrease the magnetic field of the dipole to mitigate the deleterious effect of ISR. Another option is to increase the energy modulation amplitude in modulator 2 which would reduce the optimized strength of the dispersion sections.

The ISR effect in the dispersion section 2 is negligible because of its relatively small dispersion strength and the main concern is the CSR that may result in additional energy modulation of the beam if it has a density modulation in the dispersion section. A detailed study of CSR effect is beyond the scope of this paper, and we limit our consideration here by a simple estimate. For the same configuration as that used in estimation of the ISR effect above, the second dispersion section has the bending angle of $1.2^{\circ}$ to provide a dispersion strength of $0.35 \mathrm{~mm}$. Because of the presence of $R_{51}$, the bunching is smeared out everywhere inside the chicane and it only occurs at the very end of the last dipole where $R_{51} \sigma_{x}$ is much smaller than the wavelength of the generated harmonic. Assuming the beam peak current is $800 \mathrm{~A}$ and the bunching factor 0.1 at this region and using the steady-state CSR impedance per unit length of path (see, e.g., [13])

$$
Z_{\mathrm{CSR}}(k)=(1.63+0.94 i) \frac{k_{E}^{1 / 3}}{\rho^{2 / 3}}
$$

we estimate the CSR induced slice energy to be about $1.8 \mathrm{keV}$. Considering the fact that the energy change in the 4th dipole only slightly affects the longitudinal position of the particle, the CSR effect does not seem significant to affect the bunching process. As for the first dispersion section, even though there exists a position in the second dipole where $R_{56}^{(1)}(s) \Delta E_{1} / E_{0} \approx \lambda / 4$ is satisfied, the energy modulation does not effectively convert to density modulation due to the considerable $R_{51}$ at this position and the CSR effects do not play a role.

Another issue that affects the efficacy of the echo modulation is the finite transverse size of the electron and laser beams. Because of the finite size of the laser beam, electrons with different radial positions will see the laser field of various amplitudes, which will cause a slice energy spread and may result in degradation of the density modulation. This effect is also present in the classic HGHG scheme. To minimize it, in the design of a HGHG FEL, one requires the rms laser spot size in the modulator to be much larger than that of the electron beam.

To evaluate this effect for the echo harmonic generation, we assume a Gaussian profile for the laser beam with an rms transverse size $\sigma_{r}$. We can calculate the energy modulation (expressed in units $m c^{2}$ ) for an electron located at radius $r$ using the following equation [14]: 


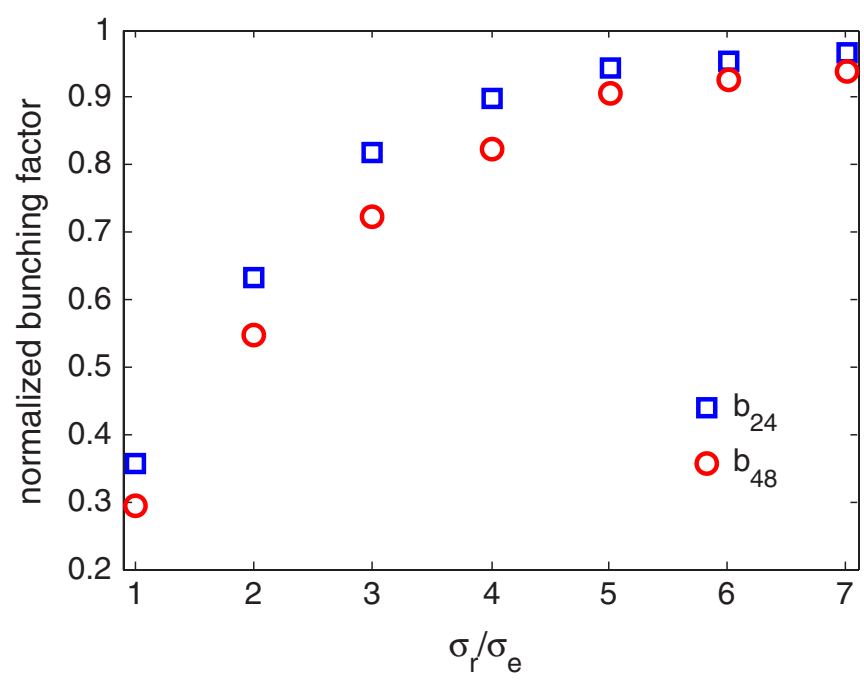

FIG. 8. (Color) Normalized bunching factors for $b_{24}$ (blue symbols) and $b_{48}$ (red circles) as functions of the ratio $\sigma_{r} / \sigma_{e}$.

$$
\begin{aligned}
\Delta \gamma(r)= & \sqrt{\frac{P_{L}}{P_{0}}} \frac{K_{u} L_{u}}{\gamma \sigma_{r}}\left[J_{0}\left(\frac{K_{u}^{2}}{4+2 K_{u}^{2}}\right)-J_{1}\left(\frac{K_{u}^{2}}{4+2 K_{u}^{2}}\right)\right] \\
& \times \exp \left(-\frac{r^{2}}{4 \sigma_{r}^{2}}\right)
\end{aligned}
$$

where $P_{L}$ is the peak laser power, $P_{0}=I_{A} m c^{2} / e \approx$ $8.7 \mathrm{GW}, L_{u}$ is the undulator length, $K_{u}$ is the undulator strength parameter, $\gamma$ is the relativistic factor for the electron beam, and $J_{0}$ and $J_{1}$ are the Bessel functions of the zeroth and first order.

We simulated the 24th harmonic bunching factor for various ratios of the laser beam size $\sigma_{r}$ to the electron beam size $\sigma_{e}$. The bunching factor for various $\sigma_{r} / \sigma_{e}$ ratios normalized to the limiting value of an infinitely large laser spot size is shown in Fig. 8. For comparison, the

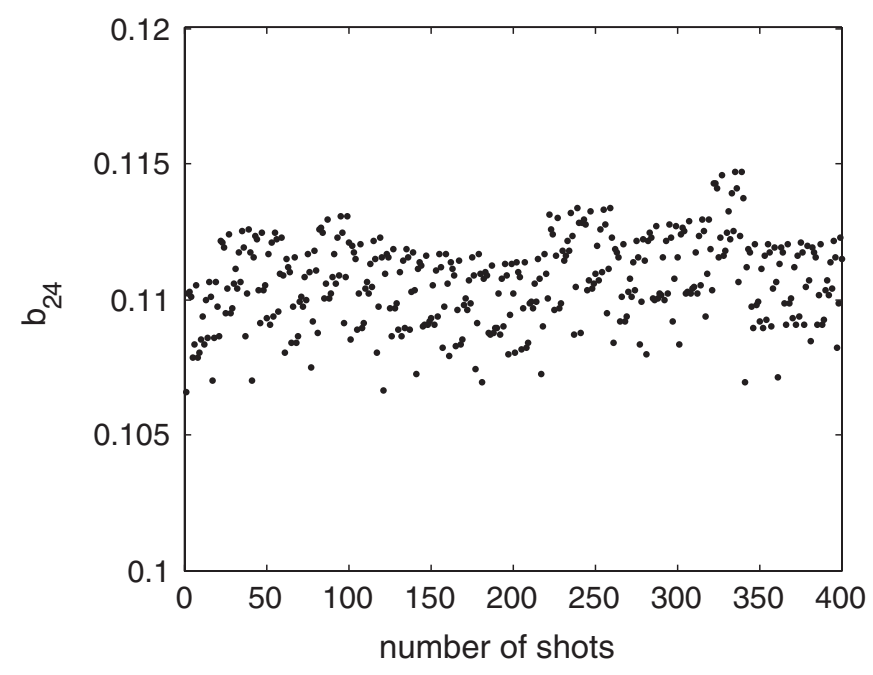

FIG. 9. Bunching factors for the 24th harmonic at various shots for a fluctuating amplitude of the laser power. normalized bunching factor for the 48th harmonic is also shown with red circles. The bunching factors for the 24th and 48th harmonics in the limit when the laser spot size tends to infinity are 0.113 and 0.088 , respectively. Note that, in order to keep the modulation amplitude the same, the laser power should scale as a square of the laser spot size. Figure 8 suggests that, to avoid a significant degradation of the EEHG FEL performance for the 24th (48th) harmonic, the rms laser size should be at least 3 times larger than that of the electron beam.

We have also studied the sensitivity of the bunching factor to the shot-to-shot fluctuations of the laser power by introducing random fluctuations of the laser power in both modulators within $\pm 5 \%$. The resulting fluctuations of the 24th harmonic are shown in Fig. 9. We see that, with the $5 \%$ tolerance on the laser peak power, the bunching factor of about 0.11 can be well maintained.

\section{PERFORMANCES OF THE EEHG FEL}

To illustrate a possible performance of the EEHG scheme we will use parameters of the Fermi@Elettra FEL project, and show how one can operate such a device at $10 \mathrm{~nm}$ wavelength. Note that the FEL-2 stage of the Fermi@Elettra project uses two-stage HGHG to generate $10 \mathrm{~nm}$ soft $\mathrm{x}$ ray seeded by a $240 \mathrm{~nm}$ laser. In the cascading HGHG scheme, the 6th harmonic $(40 \mathrm{~nm})$ of the seed laser is generated in the first stage and further used as a seed signal for the second stage. The nominal beam energy is $1.2 \mathrm{GeV}$ and local energy spread is $150 \mathrm{keV}$ [10]. Because the jitter requirement for the EEHG scheme is less stringent than that for the cascading HGHG scheme, in our calculations we take the peak current of the beam to be $800 \mathrm{~A}$, in accordance with the parameters of one stage HGHG FEL project.

The energy modulation and dispersion strengths in our example were chosen to be the same as described in Sec. III to maximize the bunching factor for the 24th harmonic. The first modulator was chosen to be $135 \mathrm{~cm}$ long with the undulator period length of $15 \mathrm{~cm}$. The input laser had a waist of 310 microns and the peak power of $64 \mathrm{MW}$. The corresponding energy modulation amplitude was $A_{1}=3$. The second modulator was $45 \mathrm{~cm}$ long and had just three undulator periods. The laser parameters were the same and the corresponding normalized energy modulation amplitude was $A_{2}=1$.

The simulation was performed with the upgraded code GENESIS $[15,16]$ and consisted of three separate runs. In the first run, the energy modulation from the $240 \mathrm{~nm}$ seed laser in the first modulator was simulated and the particle distribution was dumped at the exit of modulator 1 . The particle distribution was imported, transported through dispersion section 1, and further sent to modulator 2 for the other energy modulation. At the exit of modulator 2, the particle distribution was dumped again. Finally, the particle distribution was reimported for the third run and 

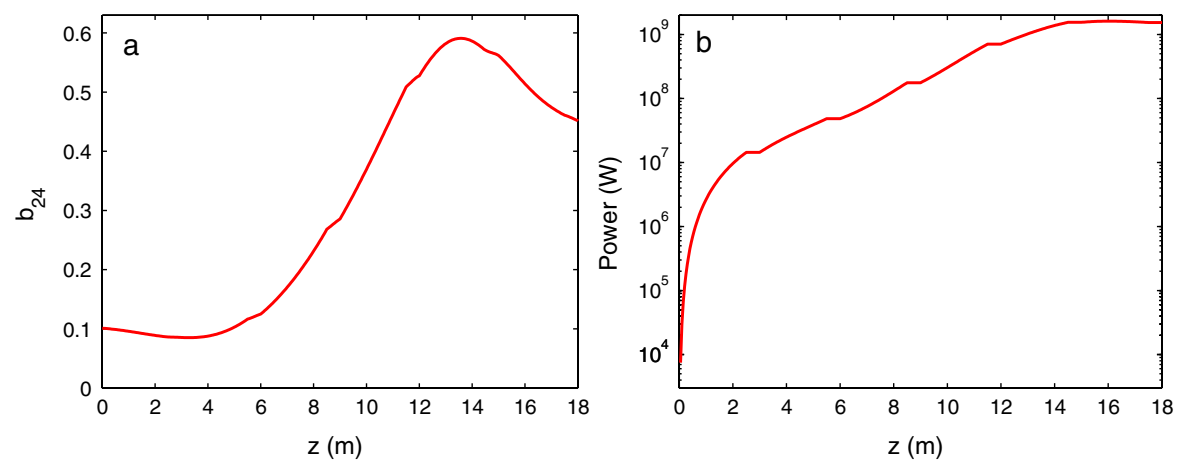

FIG. 10. (Color) (a) Bunching factor vs radiator distance for the $10 \mathrm{~nm}$ radiation. (b) Peak power vs radiator distance for the $10 \mathrm{~nm}$ radiation.

the undulator period of the radiator was tuned to the 24th harmonic of the seed laser. The radiator undulator had a period of $5 \mathrm{~cm}$ and was divided into six sections of $2.5 \mathrm{~m}$ separated by $0.5 \mathrm{~m}$ drift for focusing and beam diagnostics.

During the simulation, the dispersion strength and power of the seed laser were finely tuned to maximize the bunching factor for the 24th harmonic at the entrance to the radiator. The evolution of bunching factor and radiation power are shown in Fig. 10. The significant enhancement of the performance using the EEHG scheme is clearly seen in Fig. 10(b) where the peak power of the 24th harmonic radiation exceeds $1.6 \mathrm{GW}$ and it saturates after five undulator sections (the total magnet length is $12.5 \mathrm{~m}$ ). The large bunching factor at the entrance to the radiator offered by the EEHG scheme is responsible for the initial steep quadratic growth of the power. The high peak power and short saturation length should be attributed to the initial large bunching factor and the small energy modulation in the modulators. Indeed, the local energy spread at the entrance to the radiator is only about 2.45 times larger than the initial local energy spread.

With the same beam and laser parameters, we also simulated EEHG performance at the 48th harmonic, with the radiation wavelength of $5 \mathrm{~nm}$. In order to avoid large
ISR effects in the first dispersion section, we raised the value of $A_{2}$ to 2 by increasing the length of the modulator 2 to $90 \mathrm{~cm}$. As it follows from Eq. (7) and (8), simultaneous increase of $A_{2}$ and the harmonic number $m$ does not change much the optimal value of the parameter $B_{1}$, and hence does not increase the strength of the first dispersion section.

We first simulated the phase space evolution for the 48th harmonic with our 1D code. The longitudinal phase space at the exit of the dispersion section 2 is shown in Fig. 11(a), and the calculated bunching factors for various harmonics are shown in Fig. 11(b). We found that the 1D code gives the maximized bunching factor for the 48th harmonic about 0.088 . In a GENESIS simulation, after additional tuning of the strength of the dispersion section 2 , we found a somewhat smaller bunching factor of about 0.07 . The slight difference between the bunching factors in the GENESIS simulation and that in our 1D code is probably due to the finite laser spot size effect in the modulators. The beam with the modulation at the 48th harmonic was then sent to the radiator in which the undulator period was tuned to the $5 \mathrm{~nm}$ wavelength by shortening the period by a factor of 2 with other parameters being the same. The simulated bunching factor and power evolution along the radiator from GENESIS is shown in Fig. 12. From Fig. 12 we see
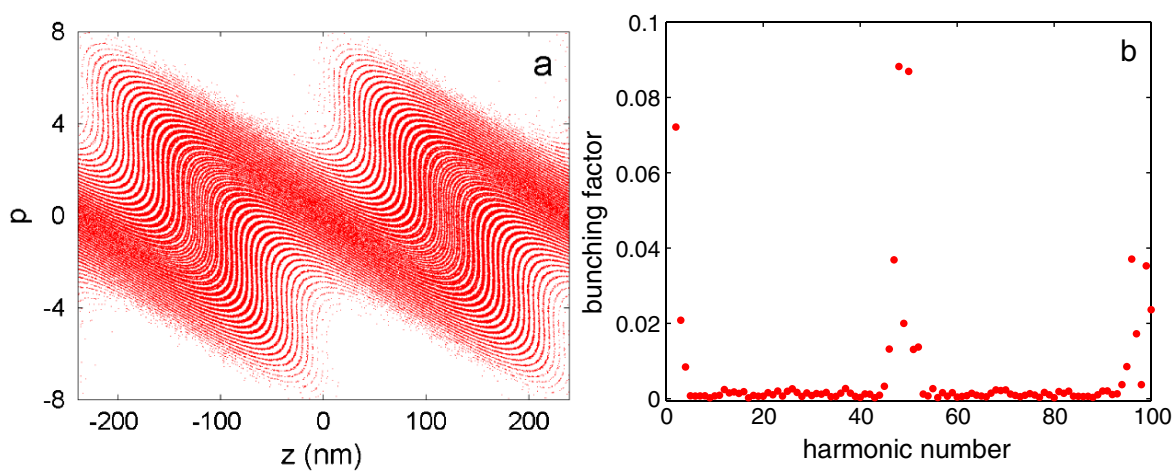

FIG. 11. (Color) (a) Longitudinal phase space at the exit of dispersion 2. (b) Bunching factors for various harmonics. 

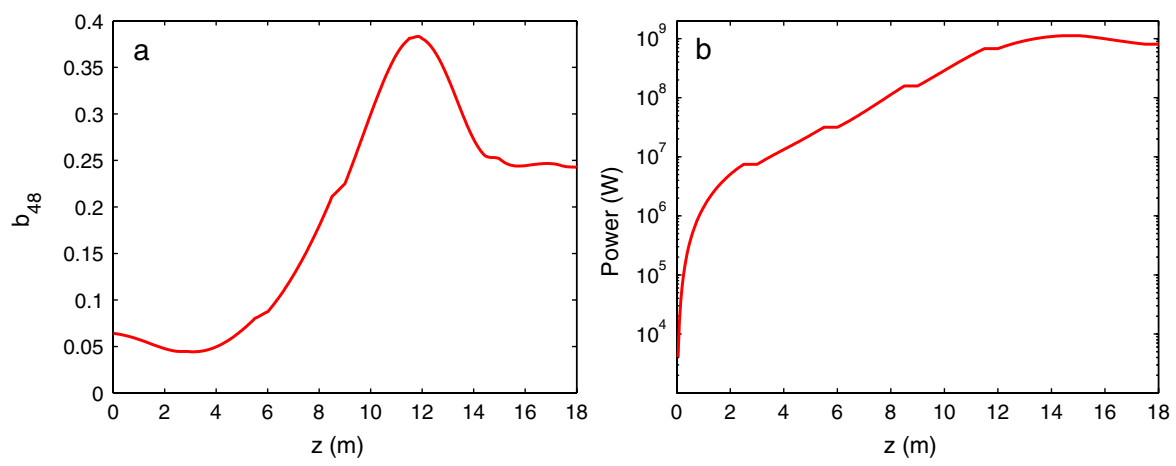

FIG. 12. (Color) (a) Bunching factor vs radiator distance for the $5 \mathrm{~nm}$ radiation. (b) Peak power vs radiator distance for the $5 \mathrm{~nm}$ radiation.

that the peak power for the $5 \mathrm{~nm}$ radiation is about $1.1 \mathrm{GW}$ and the power saturates after five undulator sections of $2.5 \mathrm{~m}$.

\section{CONCLUSIONS}

We have demonstrated a new working scheme of harmonic generation in FELs. The EEHG FEL significantly improves the performance of single-stage harmonic generation FEL. Using the realistic beam parameters (beam energy: $1.2 \mathrm{GeV}$; slice energy spread: $150 \mathrm{keV}$; peak current: $800 \mathrm{~A}$; normalized emittance: $1.5 \mathrm{~mm} \mathrm{mrad)}$ ), we have shown that $5 \mathrm{~nm}$ coherent soft $\mathrm{x}$ ray with peak power exceeding $1 \mathrm{GW}$ can be generated directly from the $240 \mathrm{~nm}$ seeding laser using the EEHG scheme. It is worth pointing out that the parameters used in our simulations and calculations are representative rather than fully optimized design sets. A more careful optimization might lead to further improvements of the scheme.

In addition to generation of high harmonics, the EEHG FEL also offer a flexibility of adjusting the x-ray pulse duration by simply adjusting the overlapping region of the two lasers. This may allow the generation of ultrashort $(<10$ fs) $x$-ray pulse with the EEHG scheme that could open up investigations of many new areas of sciences.

\section{ACKNOWLEDGMENTS}

We thank A. Chao, Y. Ding, Z. Huang, and J. Wu for helpful discussions and comments. Special thanks should be given to Y.D. and Z. H. for assistance in ELEGANT and GENESIS simulations. This work was supported by U.S. DOE Contract No. DE-AC02-76SF00515.

\section{APPENDIX}

Using notation introduced in Sec. II, we write the phase space variables transformation for the passage through the first modulator and dispersion section 1 as follows:

$$
p^{\prime}=p+A_{1} \sin \zeta, \quad \zeta^{\prime}=\zeta+B_{1} p^{\prime}
$$

where $\zeta, p$ are the initial coordinates before the entrance to the system, and $\zeta^{\prime}, p^{\prime}$ are the final coordinates at the exit from the first dispersion section. Similarly, for the passage through the second modulator and dispersion section 2 we have

$$
p^{\prime \prime}=p^{\prime}+A_{2} \sin \left(K \zeta^{\prime}+\phi\right), \quad \zeta^{\prime \prime}=\zeta^{\prime}+B_{2} p^{\prime \prime},
$$

where $\zeta^{\prime \prime}, p^{\prime \prime}$ are the space phase variables at the exit from the second dispersion section. Expressing the initial coordinate $p$ through the final variables $\zeta^{\prime \prime}$ and $p^{\prime \prime}$ and substituting it into the initial distribution function $f_{0}(p)$, we obtain the final distribution function given by Eq. (2).

The bunching factor is defined by Eq. (3):

$$
\begin{aligned}
b & =\frac{1}{N_{0}}\left|\left\langle e^{-i a \zeta} N(\zeta)\right\rangle\right| \\
& =\frac{1}{N_{0}}\left|\int_{-\infty}^{\infty} d p^{\prime \prime}\left\langle e^{-i a \zeta^{\prime \prime}} f_{f}\left(\zeta^{\prime \prime}, p^{\prime \prime}\right)\right\rangle\right|,
\end{aligned}
$$

where in the last integral we use the double prime variables to emphasize that these are the phase space variables in the final state of the beam, after the passage through the system. Unfortunately, the direct integration in Eq. (A3) cannot be carried out analytically.

There is an alternative way to calculate the bunching factor which leads to relatively simple expressions. It is based on observation that the averaging in Eq. (A3) can be understood as

$$
\langle\cdots\rangle=\lim _{L \rightarrow \infty} \frac{1}{2 L} \int_{-L}^{L} d \zeta^{\prime \prime}\langle\cdots\rangle .
$$

If we now transform from the final variables $\zeta^{\prime \prime}, p^{\prime \prime}$ to the initial variables $\zeta$ and $p$, using the fact that $f_{f}\left(\zeta^{\prime \prime}, p^{\prime \prime}\right)=$ $f_{0}(p)$ and $d \zeta^{\prime \prime} d p^{\prime \prime}=d \zeta d p$, we obtain

$$
b=\frac{1}{N_{0}}\left|\int_{-\infty}^{\infty} d p f_{0}(p)\left\langle e^{-i a \zeta^{\prime \prime}(\zeta, p)}\right\rangle\right|,
$$

where $\zeta^{\prime \prime}$ is expressed in terms of $\zeta$ and $p$, and the angular brackets are understood as averaging over $\zeta$ :

$$
\langle\cdots\rangle=\lim _{L \rightarrow \infty} \frac{1}{2 L} \int_{-L}^{L} d \zeta\langle\cdots\rangle .
$$


The expression for $\zeta^{\prime \prime}$ in terms of $\zeta$ and $p$ can be found from Eqs. (A1) and (A2):

$$
\begin{aligned}
\zeta^{\prime \prime}= & \zeta+\left(B_{1}+B_{2}\right) p+A_{1}\left(B_{1}+B_{2}\right) \sin \zeta \\
& +A_{2} B_{2} \sin \left(K \zeta+K B_{1} p+K A_{1} B_{1} \sin \zeta+\phi\right) .
\end{aligned}
$$

Substituting this expression into Eq. (A5) gives

$$
\begin{aligned}
b= & \frac{1}{N_{0}} \mid \int_{-\infty}^{\infty} d p e^{-i a p\left(B_{1}+B_{2}\right)} f_{0}(p)\left\langle e^{-i a \zeta} e^{-i a A_{1}\left(B_{1}+B_{2}\right) \sin \zeta}\right. \\
& \left.\times e^{-i a A_{2} B_{2} \sin \left(K \zeta+K B_{1} p+K A_{1} B_{1} \sin \zeta+\phi\right)}\right\rangle \mid .
\end{aligned}
$$

Two exponential factors in this equation can be expanded in series:

$$
e^{-i a A_{1}\left(B_{1}+B_{2}\right) \sin \zeta}=\sum_{k=-\infty}^{\infty} e^{i k \zeta} J_{k}\left[-a A_{1}\left(B_{1}+B_{2}\right)\right],
$$

and

$$
\begin{aligned}
& e^{-i a A_{2} B_{2} \sin \left(K \zeta+K B_{1} p+K A_{1} B_{1} \sin \zeta+\phi\right)} \\
& \quad=\sum_{m=-\infty}^{\infty} e^{i m\left(K \zeta+K B_{1} p+K A_{1} B_{1} \sin \zeta+\phi\right)} J_{m}\left(-a A_{2} B_{2}\right),
\end{aligned}
$$

and then in turn the factor $e^{i m K A_{1} B_{1} \sin \zeta}$ appearing on the right-hand side of Eq. (A10) can also be expanded:

$$
e^{i A_{1} B_{1} K m \sin \zeta}=\sum_{l=-\infty}^{\infty} e^{i l \zeta} J_{l}\left(A_{1} B_{1} K m\right) .
$$

Now collecting all the terms in (A8) that have $\zeta$ dependence, we find that they are given by the following expression:

$$
\left\langle e^{i(k+l+m K-a) \zeta}\right\rangle .
$$

Recalling that the angular brackets denote averaging over $\zeta$, we conclude that the above expression does not vanish only if

$$
a=n+m K,
$$

where $n=k+l$ is an integer, in which case the averaged value (A12) is equal to 1. Hence we obtain Eq. (4). Substituting Eqs. (A9)-(A11) into Eq. (A8) and carrying out integration over $p$ with the help of

$$
\begin{aligned}
& \frac{1}{N_{0}} \int_{-\infty}^{\infty} d p e^{-i a p\left(B_{1}+B_{2}\right)+i m K p B_{1}} f_{0}(p) \\
& \quad=e^{-(1 / 2)\left[a p\left(B_{1}+B_{2}\right)-m K p B_{1}\right]^{2}}
\end{aligned}
$$

and also using the identity

$$
J_{s}(a+b)=\sum_{k=-\infty}^{\infty} J_{k}(b) J_{s-k}(a),
$$

we arrive at Eq. (5).

[1] C. Pellegrini, in Proceedings of the 10th European Particle Accelerator Conference, Edinburgh, Scotland, 2006 (EPS-AG, Edinburgh, Scotland, 2006), pp. 36363640.

[2] A. Kondratenko and E. Saldin, Part. Accel. 10, 207 (1980).

[3] R. Bonifacio, C. Pellegrini, and L.M. Narducci, Opt. Commun. 50, 373 (1984).

[4] R. Bonifacio, L.D.S. Souza, P. Pierini, and E.T. Scharlemann, Nucl. Instrum. Methods Phys. Res., Sect. A 296, 787 (1990).

[5] L. Yu, Phys. Rev. A 44, 5178 (1991).

[6] J. Wu and L. H. Yu, Nucl. Instrum. Methods Phys. Res., Sect. A 475, 104 (2001).

[7] E. Allaria and G. D. Ninno, Phys. Rev. Lett. 99, 014801 (2007).

[8] G. Stupakov, Phys. Rev. Lett. 102, 074801 (2009).

[9] M. Abramovitz and I. A. Stegun, Handbook of Mathematical Functions (Dover, New York, 1968).

[10] Fermi@Elettra conceptual design report, http://www. elettra.trieste.it/FERMI, Elettra (2007).

[11] A. W. Chao and M. Tigner, Handbook of Accelerator Physics and Engineering (World Scientific, Singapore, 2006), 3rd ed.

[12] M. Borland, Argonne National Laboratory Technical Report LS-287, 2000.

[13] G. Stupakov and S. Heifets, Phys. Rev. ST Accel. Beams 5, 054402 (2002).

[14] Z. Huang, M. Borland, P. Emma, J. Wu, C. Limborg, G. Stupakov, and J. Welch, Phys. Rev. ST Accel. Beams 7, 074401 (2004).

[15] S. Reiche, Nucl. Instrum. Methods Phys. Res., Sect. A 429, 243 (1999).

[16] S. Reiche, P. Musumeci, and K. Goldammer, in Proceedings of the 2007 Particle Accelerator Conference, Albuquerque, New Mexico, 2007 (IEEE, Albuquerque, New Mexico, 2007). 